\title{
Clinical outcomes of personalized frozen-thawed embryo transfer timing for patients with recurrent implantation failure
}

\author{
Lifei Li ${ }^{1,2 \#}$, Zhijian Kou ${ }^{3 \#}$, Yujie Fu ${ }^{3}$, Lanlan Liang ${ }^{1}$, Lin Liu ${ }^{2}$, Xuehong Zhang ${ }^{1,2}$ \\ ${ }^{1}$ Reproductive Medicine Center of the First Hospital of Lanzhou University, Lanzhou, China; ${ }^{2}$ Key Laboratory for Reproductive Medicine and \\ Embryo of Gansu, Lanzhou, China; ${ }^{3}$ Department of Anesthesiology, Gansu Provincial Hospital, Lanzhou, China \\ Contributions: (I) Conception and design: L Li; (II) Administrative support: None; (III) Provision of study materials or patients: L Li; (IV) Collection \\ and assembly of data: Y Fu, Z Kou; (V) Data analysis and interpretation: L Liang, L Liu; (VI) Manuscript writing: All authors; (VII) Final approval of \\ manuscript: All authors. \\ "These authors contributed equally to this work. \\ Correspondence to: Xuehong Zhang, MD. \#1 Donggang West Road, Lanzhou 730000, China. Email: zhangxueh@lzu.edu.cn.
}

\begin{abstract}
Background: Recurrent implantation failure (RIF) is a critical problem for assisted reproduction technology. High-quality embryos and the synchronization endometrium both have great significance. How to get the optimal endometrial receptivity is a challenge for implantation and pregnancy of infertile patients with RIF. The objective of this study is to investigate personalized protocol of frozen-thawed embryo transfer (FET) cycles, and its effect on clinical outcomes in patients with RIF.

Methods: We chose 91 RIF patients from January 2017 to June 2019 in the Reproductive Medicine Center of the First Hospital of Lanzhou University. A total of 100 FET cycles were undertaken with a gonadotropinreleasing hormone agonist (GnRH-agonist) protocol combined with hormone replacement therapy (HRT) for endometrial preparation. The patients were divided into two groups: the routine group (cleavage embryo transferred at day 3 after luteal support) included 48 cycles; the personalized group included 52 cycles with delayed endometrial trigger and luteal support (the time of embryo transfer depended on the level of serum hormone and endometrial thickness).

Results: The data showed the personalized group had longer time for endometrial preparation. On the day of embryo transfer, serum progesterone $(\mathrm{P})$ and the $\mathrm{E}_{2} / \mathrm{P}$ ratio was significantly different compared with the routine group $(\mathrm{P}<0.05)$. The clinical pregnancy rate in the routine group was $35.42 \%(17 / 48)$ and $59.62 \%$ $(31 / 52)$ in the personalized group $(\mathrm{P}<0.05)$. The abortion rate was not significantly different.
\end{abstract}

Conclusions: For women with RIF, personalized timing for transfer of FET resulted in a higher clinical pregnancy rate compared with routine protocol.

Keywords: Recurrent implantation failure (RIF); embryo implantation; Frozen-thawed embryo transfer (FET); personalized treatment

Submitted Oct 21, 2021. Accepted for publication Jan 18, 2022.

doi: $10.21037 /$ atm-22-161

View this article at: https://dx.doi.org/10.21037/atm-22-161

\section{Introduction}

Since the first success of frozen-thawed embryo transfer (FET) in 1983, it has been widely used in assisted reproduction. FET has now become a viable alternative method in vitro fertilization (IVF), which allows the storing of excess embryos, reduces multiple pregnancy rates, avoids severe ovarian hyperstimulation syndrome, and improves the effective use of embryos (1). The critical factor of FET is synchronization of blastocyst development with receptivity of the endometrium, which is controlled by estrogen $\left(\mathrm{E}_{2}\right)$ and progesterone $(\mathrm{P})(2)$. The optimal time at which the endometrium is considered to be receptive is called the window of implantation which occurs between 
days 19 and 22 in a natural 28-day cycle (3).

There is a choice of different protocols for preparation of the endometrium before FET, including natural cycles, ovarian stimulation cycles and hormone replacement therapy (HRT) cycles. HRT cycles involve the endometrium being primed by the administration of $\mathrm{E}_{2}$ and $\mathrm{P}$, while spontaneous ovulation is prevented by administration of gonadotropinreleasing hormone agonist (GnRH-agonist) (4). The cleavage embryos are usually transferred with 3 days of $\mathrm{P}$ preparation, because this protocol is considered to be synchronized with the fully developed endometrial pinopodes $(5,6)$. However, some studies have suggested that the pinopodes are not accurate markers of the implantation window (7), and the optimal implantation window of FET during a GnRH-agonist protocol combined with a HRT cycle remains controversial (8-11). Unlike fresh embryo transfer cycles, FET cycles may have a major advantage that allows to control the embryo transfer timing after preparation of the endometrium. This is likely to be of most benefit for women who have experienced recurrent implantation failure (RIF).

RIF should be considered when a woman has undergone more than three failed fresh or frozen embryo transfers with four or more good-quality embryos (cleavage embryos or blastocysts) to achieve clinical pregnancy (12). There are many reasons for RIF, which include underlying diseases of patients, uterine abnormalities, and embryonic factors (13). Recently some researchers analysed the influence of the vaginal microbiota and metabolites on RIF and firstcycle successful FET patients, and showed that the vaginal microbiota structure of the RIF group was different from that of the control (14). However, RIF can still occur even if these factors can be treated. It is now becoming accepted that the window of implantation may occur at different times in different women $(3,15)$, a personalized approach to embryo transfer has been considered according to clinical indicators $(16,17)$.

In this study, RIF patients in the Reproductive Medicine Center of the First Hospital of Lanzhou University were studied. To evaluate the effection of modified therapy, comparisons were made between patients who received routine FET involving standard 3-day transfer timing, and patients who received a personalized FET with prolonged the timing of transfer.

We present the following article in accordance with the TREND reporting checklist (available at https://atm. amegroups.com/article/view/10.21037/atm-22-161/rc).

\section{Methods}

\section{Study subjects}

From January 2017 to June 2019, in the Reproductive Medicine Center of the First Hospital of Lanzhou University, 91 RIF patients (total 100 cycles) underwent FET with GnRH-agonist protocol for endometrial preparation. All procedures performed in this study involving human participants were in accordance with the Declaration of Helsinki (as revised in 2013). The study was approved by Institutional Ethics Committee of the First Hospital of Lanzhou University (No. LDYYLL2021-410) and informed consent was taken from all the patients. The criteria for inclusion were: (I) RIF; (II) age $<38$ year; and (III) GnRH-agonist protocol combined with HRT cycle for endometrial preparation. No eligible patients were excluded. RIF was defined as at least three times previous failure of embryo transfer whether fresh or FET cycles that involved at least four or more good-quality cleavage embryos or blastocysts. The study included patients from two different periods. At first, the conventional method was used until December 2017. To improve the outcomes for these patients, we decided to delay the time of transfer that called a personalized transfer strategy. This modified method was clinically practiced for all subsequent patients (from January 2018 to June 2019). Therefore, the patients were separated into two groups according to the ET method: the routine group included 48 cycles, in which the cleavage embryos were transferred to the endometrium with 3 days of $\mathrm{P}$ preparation; the personalized group included 52 cycles, in which endometrial preparation was prolonged.

\section{Endometrial preparation}

An AlokaF75 color Doppler ultrasound scanner was used with a vaginal probe frequency of $7.5 \mathrm{MHz}$ to detect the endometrial thickness. All the ultrasound examinations were performed by the same doctor. In the routine group, all the patients underwent embryo transfer under GnRHagonist protocol combined with an HRT cycle. During the mid-luteal phase (18-22 days) before the treatment, patients received the GnRH-agonist suppression for 3-4 cycles using long-acting triptorelin $0.5 \mathrm{~mL} /$ diphereline $0.9 \mathrm{~mL}$ (triptorelin, Ferring pharmaceutical company/diphereline, Shanghai Lizhu Co., Ltd., China). At 3 weeks after the last GnRH-agonist treatment, transvaginal ultrasound was conducted. When indicators suggested that suppression 
had worked well (transvaginal ultrasound monitored the follicle diameter $<5 \mathrm{~mm}$, endometrial thickness $<5 \mathrm{~mm}$, serum $\mathrm{E}_{2}<20 \mathrm{pg} / \mathrm{mL}$ ), estradiol valerate (Progynova, German Bayer Pharmaceutical Company) was taken orally at a dose of 4-6 mg/day. After 7-8 days, the endometrial thickness was measured by ultrasound and the dosage was adjusted accordingly. After 14 days, if the endometrial thickness was $\geq 8 \mathrm{~mm}$, human chorionic gonadotropin (hCG) $5,000-10,000$ IU was injected intramuscularly. On the $3 \mathrm{rd}$ day after hCG administration, luteal support was given according to endometrial thickness. The patients underwent embryo transfer after 3 days of luteal support.

In the personalized group, the protocol of Progynova adiministration was the same as in the routine group. On the 12th day, serum $\mathrm{E}_{2}$ and luteinizing hormone $(\mathrm{LH})$ were measured daily. According to the trend of these hormones and the endometrial thickness, hCG 5,000-10,000 IU was injected and 48-72 h later, luteal support was given (when serum LH decreased and serum $P$ increased compared with the day before). After 5 days of luteal support, the embryo transfer was taken according to the endometrial thickness $(>7 \mathrm{~mm}$ ).

Blood $\beta$-hCG levels were tested 14 days after ET and transvaginal ultrasound was performed 28 days after ET to confirm the gestational sac and cardiac activity.

\section{Embryo thawing}

Embryo thawing was performed according to conventional vitrification procedures. Embryos were classified into four grades. Grade 1: blastomeres were of equal size with no cell death and fragmentation $<10 \%$; Grade 2: blastomeres were mostly of equal size, with cell death or fragmentation between 10-20\%; Grade 3: embryos fragmentation or dead blastomeres were between 20-50\%; Grade 4: embryos fragmentation or dead blastomeres were $>50 \%$. Grade 1 and 2 embryos were considered to be high-quality embryos.

Before 2019, the criteria for ET was as follows: $\geq 1$ highquality embryo were thawed, then 2 embryos were routinely transferred; if patients were over 35 years old without a high-quality thawed embryo, transfer of three embryos was considered.

\section{Statistical analysis}

SPSS 16.0 (IBM, USA) was used for statistical analysis of all the data, which underwent a normality test. If the data showed a normal distribution, it was represented as mean \pm standard deviation and a $t$-test was used. If the data showed a non-normal distribution, the Kruskal-Wallis nonparametric test was used for comparison between groups. $\mathrm{P}<0.05$ was considered statistically significant.

\section{Results}

\section{Comparison of baseline information and implantation time}

The differences in baseline information were not statistically significant in the two groups of patients $(\mathrm{P}>0.05)$, including average age, body mass index (BMI), basal ovarian function, type of infertility, and etiology of infertility (Table 1).

The differences between the two groups in terms of the implantation time were shown in Table 2. The time from hCG administration to luteal support was adjusted from 3 days to 4 days in 15 of the 52 cycles $(28.85 \%)$ in the personalized group and the time from luteal support to embryo transfer was prolonged to 5 days for the majority of the women in the personalized group compared with the routine group.

\section{Comparison of hormone levels on the day of embryo transfer and clinical outcomes}

The two groups were similar in terms of the number of transferred embryos, number of transferred high-quality embryos, and endometrial thickness on the day of embryo transfer $(\mathrm{P}>0.05$, Table 3$)$. Compared with the routine group, the serum $\mathrm{P}$ on the day of $\mathrm{ET}$ was significantly different $(19.01 \pm 8.95$ vs. $13.92 \pm 2.97, \mathrm{P}=0.000)$, and the $\mathrm{E}_{2} / \mathrm{P}$ ratio was higher $(\mathrm{P}<0.05)$, but there was no differences of the serum $\mathrm{E}_{2}$ on the day of $\mathrm{ET}(\mathrm{P}>0.05)$. The clinical pregnancy rate in the routine group was $35.42 \%$ (17/48), and the abortion rate was $23.53 \%$ (4/17). The clinical pregnancy rate of $59.62 \%(31 / 52)$ in the personalized group was significantly higher than that of the routine group $(\mathrm{P}<0.05)$, but the abortion rate was $19.35 \%(6 / 31)$, which was not significantly different $(\mathrm{P}>0.05)$.

\section{Discussion}

RIF is a common clinical problem that is very difficult to treat. Our center defined it as non-pregnancy after at least three failed fresh or frozen embryo transfers with at least four or more good-quality embryos. The occurrence of RIF involves the quality of embryos, endometrial receptivity, autoimmunity and other factors (13). Therefore, high- 
Table 1 Baseline information of patients in the two FET protocol groups

\begin{tabular}{|c|c|c|c|}
\hline Characteristics & Routine group $(n=42)$ & Personalized group $(n=49)$ & $P$ value \\
\hline \multicolumn{4}{|l|}{ Clinical features } \\
\hline Cycles, $\mathrm{n}$ & 48 & 52 & \\
\hline Age, years & $32.79 \pm 2.80$ & $32.02 \pm 3.89$ & 0.291 \\
\hline $\operatorname{BMI}\left(\mathrm{kg} / \mathrm{m}^{2}\right)$ & $22.42 \pm 2.22$ & $22.00 \pm 2.32$ & 0.383 \\
\hline $\mathrm{bLH}(\mathrm{mlU} / \mathrm{mL})$ & $4.93 \pm 4.34$ & $5.14 \pm 3.70$ & 0.818 \\
\hline $\mathrm{bE}_{2}(\mathrm{pg} / \mathrm{mL})$ & $53.91 \pm 21.80$ & $42.60 \pm 27.90$ & 0.202 \\
\hline Mean No. of previous ETs & $4.02 \pm 1.26$ & $3.76 \pm 1.11$ & 0.282 \\
\hline \multicolumn{4}{|l|}{ Type of infertility } \\
\hline \multicolumn{4}{|l|}{ Cause of infertility } \\
\hline Endometritis & $5(11.90 \%)$ & $7(14.29 \%)$ & 0.738 \\
\hline Endometrial polyp & $5(11.90 \%)$ & $4(8.16 \%)$ & 0.728 \\
\hline Hysteromyoma & $2(4.76 \%)$ & $2(4.08 \%)$ & 1.000 \\
\hline Intrauterine adhesions & $3(7.14 \%)$ & $1(2.04 \%)$ & 0.332 \\
\hline thin endometrial lining & $4(9.52 \%)$ & $5(10.20 \%)$ & 1.000 \\
\hline Endometriosis & $2(4.76 \%)$ & $4(8.16 \%)$ & 0.683 \\
\hline PCOS & $5(11.90 \%)$ & $6(12.24 \%)$ & 0.960 \\
\hline
\end{tabular}

FET, frozen-thawed embryo transfer; BMI, body mass index; bFSH, basal follicle-stimulating hormone; bLH, basal luteinizing hormone; $\mathrm{bE}_{2}$, basal estrogen; ET, embryo transfer; PCOS, polycystic ovary syndrome.

Table 2 Implantation time of patients in the two FET protocol groups

\begin{tabular}{lcc} 
& Routine group (48 cycles) & Personalized group (52 cycles) \\
\hline Time from hCG administration to luteal support & 48 & 36 \\
3 days & - & 15 \\
4 days & - & 1 \\
5 days & & 0 \\
Time from luteal support to embryo transfer & 1 & 2 \\
3 days & 43 & 48 \\
4 days & 4 & 2 \\
5 days & 0 & \\
\hline
\end{tabular}

FET, frozen-thawed embryo transfer; hCG, human chorionic gonadotropin. 
Table 3 Clinical outcomes of patients in the two FET protocol groups

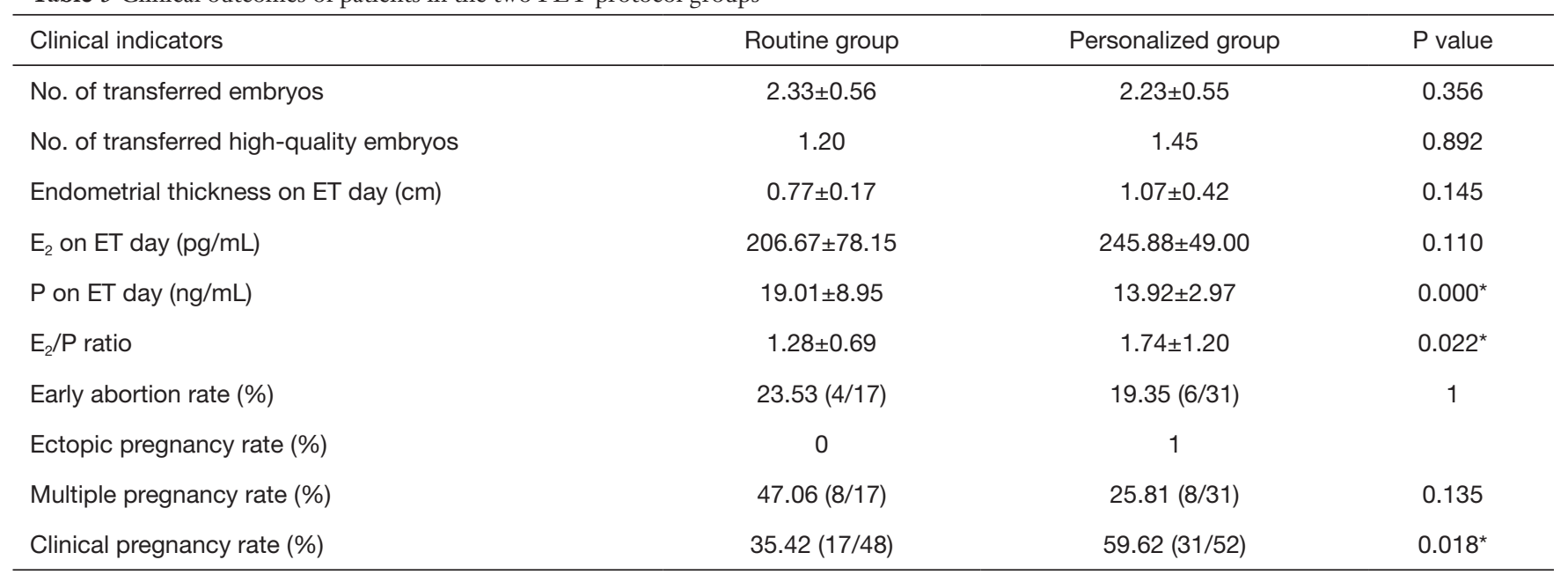

${ }^{*}, \mathrm{P}<0.05$. FET, frozen-thawed embryo transfer; $\mathrm{E}_{2}$, estrogen; ET, embryo transfer; P, progesterone.

quality embryos and the appropriate endometrial preparation protocol both have great significance for improving the FET pregnancy rate. The aim of this study was to investigate whether personalized treatment, regarding transfer timing, would improve pregnancy outcomes in FET cycle. The results showed there was a significant improvement in pregnancy rates with the personalized approach.

Based on current studies, it can be difficult to evaluate which protocols for FET provides the best outcomes (11). Clinically, a HRT cycle is mostly used for FET patients with RIF. However, HRT cycles have various problems, such as the cancellation of transfer due to spontaneous follicular developmen. Furthermore, there is a LH peak that is similar to the endogenous peak during the estradiol valerate treatment, high LH levels may affect endometrial receptivity and reduce embryo implantation rates (18). As early as 1988, Salat-Baroux et al. (19) proposed that prior to HRT, pituitary suppression can promote the synchronized development of endometrium and embryo. Another study showed that in RIF patients diagnostic hysteroscopy combined with endometrial preparation of a GnRH-agonist protocol, can significantly increase the embryo implantation rates and clinical pregnancy rates (20). GnRH is a decapeptide compound synthesized and released by hypothalamic neurons, and directly secreted into the pituitary portal system. It is shown that $\mathrm{GnRH}$-agonist can increase the expression of endometrial integrin $a_{v} \beta_{3}$, which participates in blastocyst adhesion by mediating the interaction between trophoblast cells and endometrial epithelial cells, to improve endometrial receptivity.
Surrey et al. (21) evaluated that two months of GnRHa administration "after vitrification of all embryos in IVF patients with endometriosis and/or aberrant endometrial integrin $a_{v} \beta_{3}$ expression during the early secretory phase" led to high implantation and ongoing pregnancy rates despite a high incidence of prior cycle failure". Another study found that, by inducing endometrial urokinase type plasminogen activator, plasminogen activator inhibitor and other cellular molecules, transforming growth factor (TGF) inhibited trophoblast invasion, promoting apoptosis of decidual cells and effect the embryo implantation (22). GnRH-agonist can interfere with the TGF- $\beta$ receptor signaling pathway to affect the secretion of endometrial TGF- $\beta$ (23). Therefore, GnRH-agonist not only allows synchronization of follicular development, but also improve endometrial receptivity. In this study we administered 3-4 cycles of GnRH-agonist for patients with RIF in the mid-luteal cycle before treatment, and got better clinical outcomes.

It is well known that under normal physiological conditions hCG plays a protective role for luteal function, which continuously produces P. In general, hCG acts on the receptor of the lutein cells, activates adenylate cyclase, generates biochemical reactions to prolong luteal life and stimulates the biological synthesis of endogenous $E_{2}$ and $\mathrm{P}$ (24) to regulates the growth and development of normal embryos (25). The number of hCG receptors and affinity in the endometrium gradually increase with changes in the endometrium from the proliferative phase to the secretory phase (26). If the hCG receptors decrease in number or affinity, a series of pathological reactions occurs. LH/ 
hCG receptors are found in the myometrium and uterine vascular systems, and may promote uterine vasodilation (27), which suggests that hCG and its receptors in the endometrium are extremely important for the normal endocrine environment, that is required for maintaining embryo development in the uterus. Therefore, we speculate that endometrial morphological and physiological changes are not only controlled by steroid hormones, but also affected by hCG and its receptors. A combination of factors is required in order to maintain a normal pregnancy. Therefore, in present study we detected serum LH change and administered hCG to promote the active of secretory phase endometrium during the FET cycle, it was the same as ovulation mechanism of LH surge in natural and IVF cycle. It was intended by this "endometrium trigger" effect that the best implantation environment would be achieved, to benefit embryo implantation.

Although endometrial preparation with a HRT cycle can achieve similar clinical outcome to natural ovulation cycles (28), it is different for the patients with RIF, therefore, the window of implantation has become a concern in the HRT cycle. Similar to other studies $(16,17)$ that have also attempted personalized treatment, our results showed that the personalized group had a significant improvement in the clinical pregnancy rate compared with the routine group. When the serum $\mathrm{P}$ fluctuated between 10 and $15 \mathrm{ng} / \mathrm{mL}$ on the day of ET, the best pregnancy outcomes were achieved (29). As we know, progesterone induces differentiation of endometrial epithelial and stromal cells in the secretory phase, along with changes in the vasculature, extracellular matrix and leucocyte content of endometrium. However, in IVF cycle (1), controlled ovarian stimulation exposes the endometrium to supraphysiological concentrations of estrogen and progesterone, and premature elevation in progesterone has a negative effect on the endometrial receptivity in fresh embryo transfer cycles. One study (30) found that there were fully developed pinopodes on the endometrium of mice on the day 5 of $\mathrm{P}$ injection compared with on the day 4 , which indicated that a delay in the implantation window may have occurred in FET cycle. Those revealed both appropriate concentrations and timing of steroid hormones are critical to receptivity. So we consider there is a time delay in the implantation window in some women underwent FET with GnRH-agonist combined with a HRT cycle. This is consistent with the results of the ground research conducted by Li Ruirui (31) and the suggestion that the window of transfer may not be the same in all women
(14-16). We speculate that when the P level is $<10 \mathrm{ng} / \mathrm{mL}$, the endometrial $\mathrm{P}$ receptors are not completely bound to interfere the endometrium into the secretory phase. Meanwhile, when the $\mathrm{P}$ level is $>15 \mathrm{ng} / \mathrm{mL}$, the effect of serum $\mathrm{P}$ for endometrial receptivity will be amplified.

In addition, the data from this study showed the clinical pregnancy rates of the two groups were significantly different. Some researchers believe that the appropriate $\mathrm{E}_{2} / \mathrm{P}$ ratio determines the distribution of glycosyl conjugates on the endometrial epithelial surface, that affect embryo implantation. Therefore, for RIF patients, appropriately prolonging the time of endometrial preparation can improve endometrium receptivity and clinical pregnancy rate.

\section{Conclusions}

Personalized protocol prolonged the endometrial preparation time compared with routine protocol. On the day of ET, the $\mathrm{E}_{2} / \mathrm{P}$ ratio was significantly different between groups, that was benefit for an increased pregnancy rate. Therefore, for the women with RIF personalized timing of FET resulted in better clinical outcomes.

\section{Acknowledgments}

We wish to thank our study group for their assistance with collecting patient data and analysis.

Funding: The study was supported by Natural Science Foundation of Gansu Province (No. 21JR7RA366), and Lanzhou Chengguan District Science and Technology Project (No. 2021-9-8).

\section{Footnote}

Reporting Checklist: The authors have completed the TREND reporting checklist. Available at https://atm. amegroups.com/article/view/10.21037/atm-22-161/rc

Data Sharing Statement: Available at https://atm.amegroups. com/article/view/10.21037/atm-22-161/dss

Conflicts of Interest: All authors have completed the ICMJE uniform disclosure form (available at https://atm. amegroups.com/article/view/10.21037/atm-22-161/coif). The authors have no conflicts of interest to declare.

Ethical Statement: The authors are accountable for all aspects of the work in ensuring that questions related 
to the accuracy or integrity of any part of the work are appropriately investigated and resolved. All procedures performed in this study involving human participants were in accordance with the Declaration of Helsinki (as revised in 2013). The study was approved by Institutional Ethics Committee of the First Hospital of Lanzhou University (No. LDYYLL2021-410) and informed consent was taken from all the patients.

Open Access Statement: This is an Open Access article distributed in accordance with the Creative Commons Attribution-NonCommercial-NoDerivs 4.0 International License (CC BY-NC-ND 4.0), which permits the noncommercial replication and distribution of the article with the strict proviso that no changes or edits are made and the original work is properly cited (including links to both the formal publication through the relevant DOI and the license). See: https://creativecommons.org/licenses/by-nc-nd/4.0/.

\section{References}

1. Evans J, Hannan NJ, Edgell TA, et al. Fresh versus frozen embryo transfer: backing clinical decisions with scientific and clinical evidence. Hum Reprod Update 2014;20:808-21.

2. Valdes CT, Schutt A, Simon C. Implantation failure of endometrial origin: it is not pathology, but our failure to synchronize the developing embryo with a receptive endometrium. Fertil Steril 2017;108:15-8.

3. Lessey BA, Young SL. What exactly is endometrial receptivity? Fertil Steril 2019;111:611-7.

4. Groenewoud ER, Cohlen BJ, Al-Oraiby A, et al. A randomized controlled, non-inferiority trial of modified natural versus artificial cycle for cryo-thawed embryo transfer. Hum Reprod 2016;31:1483-92.

5. Konc J, Kanyo K, Varga E, et al. The effect of cycle regimen used for endometrium preparation on the outcome of day 3 frozen embryo transfer cycle. Fertil Steril 2010;94:767-8.

6. Rarani FZ, Borhani F, Rashidi B. Endometrial pinopode biomarkers: Molecules and microRNAs. J Cell Physiol 2018;233:9145-58.

7. Quinn KE, Matson BC, Wetendorf M, et al. Pinopodes: Recent advancements, current perspectives, and future directions. Mol Cell Endocrinol 2020;501:110644.

8. Ghobara T, Gelbaya TA, Ayeleke RO. Cycle regimens for frozen-thawed embryo transfer. Cochrane Database Syst Rev 2017;7:CD003414.
9. Kang J, Park J, Chung D, et al. Comparison of the clinical outcome of frozen-thawed embryo transfer with and without pretreatment with a gonadotropin-releasing hormone agonist. Obstet Gynecol Sci 2018;61:489-96.

10. Sahin G, Acet F, Calimlioglu N, et al. Live birth after frozen-thawed embryo transfer: which endometrial preparation protocol is better? J Gynecol Obstet Hum Reprod 2020;49:101782.

11. Cédrin-Durnerin I, Isnard T, Mahdjoub S, et al. Serum progesterone concentration and live birth rate in frozenthawed embryo transfers with hormonally prepared endometrium. Reprod Biomed Online 2019;38:472-80.

12. Bashiri A, Halper KI, Orvieto R. Recurrent Implantation Failure-update overview on etiology, diagnosis, treatment and future directions. Reprod Biol Endocrinol 2018; $16: 121$.

13. Bord I, Tamir B, Harlev A, et al. Recurrent implantation failure in IVF: features of cycles that eventually ended in conception. Arch Gynecol Obstet 2016;293:893-900.

14. Fu M, Zhang $X$, Liang $Y$, et al. Alterations in Vaginal Microbiota and Associated Metabolome in Women with Recurrent Implantation Failure. mBio 2020;11:03242-19.

15. Ruiz-Alonso M, Blesa D, Díaz-Gimeno P, et al. The endometrial receptivity array for diagnosis and personalized embryo transfer as a treatment for patients with repeated implantation failure. Fertil Steril 2013;100:818-24.

16. Ruiz-Alonso M, Galindo N, Pellicer A, et al. What a difference two days make: "personalized" embryo transfer (pET) paradigm: a case report and pilot study. Hum Reprod 2014;29:1244-7.

17. Yang S, Pang T, Li R, et al. The individualized choice of embryo transfer timing for patients with elevated serum progesterone level on the HCG day in IVF/ICSI cycles: a prospective randomized clinical study. Gynecol Endocrinol 2015;31:355-8.

18. Burks H, Paulson R. Cryopreserved embryo transfer: endometrial preparation and timing. Semin Reprod Med 2015;33:145-52.

19. Salat-Baroux J, Cornet D, Alvarez S, et al. Pregnancies after replacement of frozen-thawed embryos in a donation program. Fertil Steril 1988;49:817-21.

20. Rigos I, Athanasiou V, Vlahos N, et al. The Addition of Endometrial Injury to Freeze-All Strategy in Women with Repeated Implantation Failures. J Clin Med 2021;10:2162.

21. Surrey ES, Minjarez DA, Schoolcraft WB. The incidence of aberrant endometrial alphavbeta(3) vitronectin expression in a high risk infertility population: could 
prolonged $\mathrm{GnRH}$ agonist therapy play a role? J Assist Reprod Genet 2007;24:553-6.

22. Ni N, Li Q. TGF $\beta$ superfamily signaling and uterine decidualization. Reprod Biol Endocrinol 2017;15:84.

23. Mabuchi Y, Yamoto M, Minami S, et al.

Immunohistochemical localization of inhibin and activin subunits, activin receptors and Smads in ovarian endometriosis. Int J Mol Med 2010;25:17-23.

24. Bildik G, Akin N, Esmaeilian Y, et al. hCG Improves Luteal Function and Promotes Progesterone Output through the Activation of JNK Pathway in the Luteal Granulosa Cells of the Stimulated IVF Cycles $†$. Biol Reprod 2020;102:1270-80.

25. Gridelet V, Perrier d'Hauterive S, Polese B, et al. Human Chorionic Gonadotrophin: New Pleiotropic Functions for an "Old" Hormone During Pregnancy. Front Immunol 2020;11:343.

26. Sacchi S, Sena P, Degli Esposti C, et al. Evidence for expression and functionality of $\mathrm{FSH}$ and $\mathrm{LH} / \mathrm{hCG}$ receptors in human endometrium. J Assist Reprod Genet 2018;35:1703-12.

27. Koch Y, Wimberger P, Grümmer R. Human chorionic

Cite this article as: Li L, Kou Z, Fu Y, Liang L, Liu L, Zhang X. Clinical outcomes of personalized frozen-thawed embryo transfer timing for patients with recurrent implantation failure. Ann Transl Med 2022;10(3):131. doi: 10.21037/atm-22161 gonadotropin induces decidualization of ectopic human endometrium more effectively than forskolin in an invivo endometriosis model. Exp Biol Med (Maywood) 2018;243:953-62.

28. Yarali H, Polat M, Mumusoglu S, et al. Preparation of endometrium for frozen embryo replacement cycles: a systematic review and meta-analysis. J Assist Reprod Genet 2016;33:1287-304.

29. Labarta E, Mariani G, Holtmann N, et al. Low serum progesterone on the day of embryo transfer is associated with a diminished ongoing pregnancy rate in oocyte donation cycles after artificial endometrial preparation: a prospective study. Hum Reprod 2017;32:2437-42.

30. Long Y, Wang YC, Yuan DZ, et al. GLUT4 in Mouse Endometrial Epithelium: Roles in Embryonic Development and Implantation. Front Physiol 2021;12:674924.

31. Li RR, Dong YZ, Guo YH, et al. Comparative study of pregnancy outcomes between day 3 embryo transfer and day 5 blastocyst transfer in patients with progesterone elevation. J Int Med Res 2013;41:1318-25. 